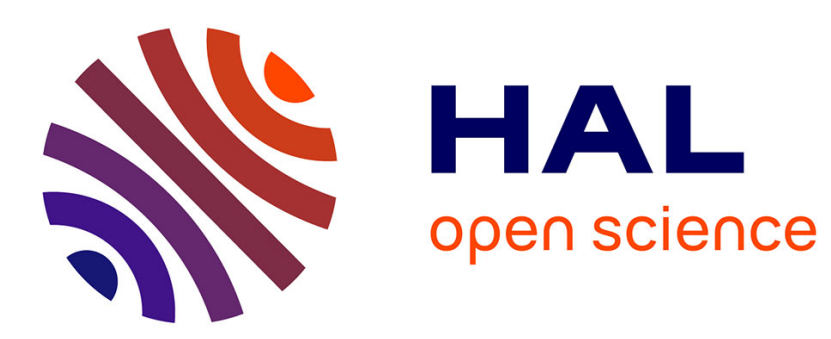

\title{
Nonlinearly generated advanced pulse waveforms for optical signal processing
}

Sonia Boscolo, Christophe Finot

\section{To cite this version:}

Sonia Boscolo, Christophe Finot. Nonlinearly generated advanced pulse waveforms for optical signal processing. 14th International Conference on Transparent Optical Networks, Jul 2012, Coventry, United Kingdom. pp.We.D1.1, 10.1109/ICTON.2012.6253925 . hal-00686332

\section{HAL Id: hal-00686332 \\ https://hal.science/hal-00686332}

Submitted on 16 Jul 2012

HAL is a multi-disciplinary open access archive for the deposit and dissemination of scientific research documents, whether they are published or not. The documents may come from teaching and research institutions in France or abroad, or from public or private research centers.
L'archive ouverte pluridisciplinaire HAL, est destinée au dépôt et à la diffusion de documents scientifiques de niveau recherche, publiés ou non, émanant des établissements d'enseignement et de recherche français ou étrangers, des laboratoires publics ou privés. 


\title{
Nonlinearly Generated Advanced Pulse Waveforms for Optical Signal Processing
}

\author{
Sonia Boscolo ${ }^{1, *}$ and Christophe Finot ${ }^{2}$ \\ ${ }^{1}$ Institute of Photonic Technologies, Aston University, Birmingham, B47ET, United Kingdom \\ ${ }^{2}$ Laboratoire Interdisciplinaire Carnot de Bourgogne, UMR 6303, BP 47870, 21078 Dijon Cedex, France \\ * Corresponding author: s.a.boscolo@aston.ac.uk
}

\begin{abstract}
Nonlinear phenomena occurring in optical fibres have many attractive features and great, but not yet fully explored potential in signal processing. Here, we review recent progress on the use of fibre nonlinearities for the generation and shaping of optical pulses, and on the applications of advanced pulse waveforms in all-optical signal processing. Among other topics, we will discuss ultrahigh repetition-rate pulse sources, the generation of parabolic-shaped pulses in active and passive fibres, the generation of pulses with triangular temporal profiles, and coherent supercontinuum sources. The signal processing applications will span optical regeneration, linear distortion compensation, optical decision at the receiver in optical communication systems, spectral and temporal signal doubling, and frequency conversion.
\end{abstract}

Keywords: nonlinear fibre optics, nonlinear pulse shaping, all-optical signal processing.

\section{INTRODUCTION}

The development of new photonic technologies for data processing in the all-optical domain is a field of growing importance with a strong potential for numerous applications in such diverse areas as optical telecommunications, metrology, optical sensing, microwave engineering, image processing, and optical computing, to name a few. Advantages of processing the information in the all-optical domain include the large available bandwidth and the (potential) parallelism intrinsic to the optical approach, which translate into highprocessing speeds. The third-order optical nonlinearity in silica-based single-mode (SM) fibres is one of the most important effects that can be used for all-optical signal processing. This happens not only because the third-order nonlinearity provides ultra-fast response times in the femtosecond range, but also because it is responsible for a wide range of phenomena such as third-harmonic generation, nonlinear refraction (Kerr nonlinearity), and stimulated Raman and Brillouin scattering. The rapid recent developments of microstructured fibres with extremely small effective core areas and exhibiting enhanced nonlinear characteristics and of fibres using materials with refractive indexes higher than that of the silica glass have enabled dramatic reduction of the required fibre lengths for nonlinear processing compared to conventional fibres. Such progress has paved the way for the integration of fibre-based nonlinear processing functions on photonic chips.

In this paper, we provide a snapshot of recent results and advances in the use of nonlinear effects in optical fibres for optical waveform generation and pulse shaping, and in the applications of advanced pulse profiles in all-optical signal processing. The main attention in this paper will be focused on results obtained in our groups.

\section{OPTICAL PULSE GENERATION AND SHAPING USING FIBRE NONLINEARITIES}

Techniques for the generation, control, and manipulation of ultrashort optical pulses and specialized waveforms have become increasingly important as a scientific technique and tool. Conventional and recently developed linear solutions for picosecond and femtosecond pulse shaping in the optical domain [1] are based on the socalled Fourier-domain approach, where the spectral-domain features of the input pulse are properly manipulated to obtain the spectral profile that corresponds to the desired temporal profile. However, a linear manipulation cannot increase the pulse bandwidth, and so to create shorter pulses nonlinear effects must be used. Moreover, in those applications that require spectral narrowing, linear filtering introduces a power penalty that is at least proportional to the ratio of the target spectrum to the input one. The pulse-shaping examples discussed in this section highlight some of the advantages offered by a passive, all-optical nonlinear fibre-based approach to pulse shaping.

As a first illustration of the possible nonlinear pulse shaping mechanisms in optical fibres, we review here the generation of ultrashort pulse trains with very high repetition rates exploiting the effect of temporal pulse compression in a nonlinear and anomalously dispersive fibre medium. Several works have demonstrated the gradual transformation of a sinusoidal beat-signal into well-separated pulses, the pulse repetition rate being simply determined by the frequency separation between the two continuous-wave laser sources. More recently, this nonlinear compression effect has been observed through a multiple four-wave mixing process in a single anomalously dispersive fibre, and has been proved to be an attractive and efficient technique to generate very high-repetition rate (up to $1 \mathrm{THz}$ ) pulse trains, combining both stability and simplicity of the experimental setup, and tunability in wavelength, pulse duration and repetition rate [2, 3]. From a fundamental point of view, the 
reshaping of a sinusoidal beat-signal into ultrashort localized structures is explained in terms of the dynamics of Akhmediev breathers as a result of the formation of Peregrine solitons [4, 5].

An important example of pulse reshaping in the normal dispersion regime of a fibre that has generated a great deal of attention in the last decade, is the formation of a particular class of pulses with a parabolic intensity profile and a linear frequency chirp in normally dispersive (ND), nonlinear fibre amplifying media. These pulses, so-called "similaritons", propagate in a self-similar manner, and can tolerate strong nonlinearity without wave breaking [6]. Alongside solitons in passive fibre, similaritons in fibre amplifiers are the most well-known classes of nonlinear attractors for pulse propagation in optical fibre. Experimental demonstrations relying on amplification from either rare-earth doping [6] or Raman scattering have been achieved [7]. Experimental interest has been strongly driven by the possibility of fully cancelling the linear chirp and thus generating ultrashort high-power pulses with very low substructures [7]. Moreover, self-similar pulse evolution in the ND passive or gain fibre of a fibre laser has been observed [8,9], and leads to major performance increases in pulse energy compared to previously-studied evolutions. In addition to single-pass fibre amplifiers and lasers, similaritons can be generated in passive fibres with dispersion-decreasing profiles [10], the longitudinal decrease of the normal dispersion being formally equivalent to linear gain. A simple approach to the generation of parabolic pulses that uses progressive nonlinear pulse reshaping in a ND fibre with fixed dispersion has been demonstrated in [11]. In contrast with the asymptotic similariton solutions obtained in gain fibres, the generated parabolic waveforms represent transient states of the nonlinear pulse evolution in the passive fibre medium [12]. As such, they have a finite life distance that depends on the initial pulse features (shape, energy, and chirp profile).

In the normal dispersion regime of a passive fibre, starting from a conventional initial field distribution, e.g., from a Gaussian pulse, besides parabolic waveforms it is possible to generate other advanced field distributions, such as flat-top- and triangular-profiled pulses with a linear chirp [12]. A necessary condition for triangular pulse formation in the fibre is that the initial pulse has a nonzero, positive chirp (using the definition $i b t^{2}$ for the phase profile). These theoretical results have been confirmed experimentally by intensity and phase measurements of the generated pulses [13] (Fig. 1(b)). An alternative method for the generation of triangular-shaped pulses in a passive ND fibre is based on the progressive reshaping of initially parabolic pulses driven by the fourth-order dispersion of the fibre [14]. Furthermore, the generation of linearly chirped pulses with a triangular distribution of the intensity has been recently demonstrated numerically as a novel type of nonlinear pulse-shaping regime in a mode-locked fibre laser [15].

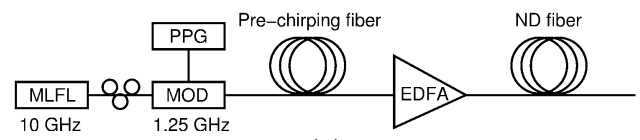

(a)

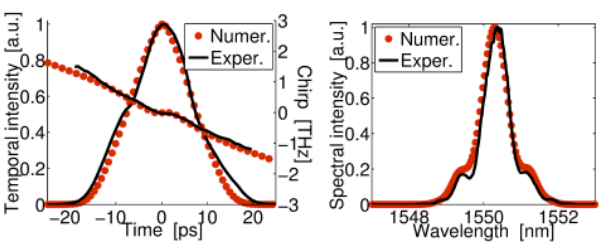

(b)

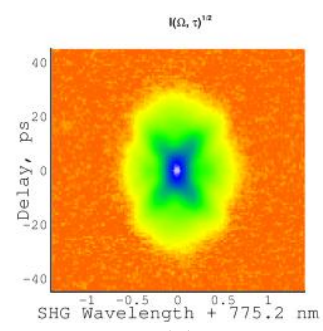

(c)

Figure 1. (a) Experimental setup for triangular pulse generation. (b) Experimental and numerical temporal intensity and chirp profiles retrieved from second-harmonic generation (SHG) frequency resolved optical gating (FROG) (left) and optical spectra (right) of the generated pulses for an example length of prechirping fibre. (c) SHG-FROG spectrogram of the pulse in (b). Results adapted from [13].

One of the most well-known examples of pulse reshaping in the spectral domain is supercontinuum generation [16]. The nonlinear pulse evolution in ND fibres leads to flatter spectra and improved temporal coherence compared to the case of continuum generated in the anomalous dispersion regime in the presence of solitonic effects and modulation instability. These features are attractive for various applications, such as in optical telecommunications where the broadened spectrum can be spectrally sliced to generate wavelength multiplexed pulse trains. The spectral ripple arising from self-phase modulation (SPM) of conventional laser pulses and the effects of optical wave breaking, which are the main limits to spectral pulse quality in the normal dispersion regime, can be avoided by using preshaped input pulses with a parabolic temporal intensity profile [17]. Low-noise multiwavelength picosecond sources covering the whole C-band of optical telecommunications have been demonstrated based on this technique. 


\section{APPLICATIONS OF PARABOLIC AND TRIANGULAR PULSES IN SIGNAL PROCESSING}

Application of parabolic pulses have not been limited to ultrashort high-power pulse generation and highly coherent continuum sources, but several optical processing techniques have taken advantage of their specific features [7]. In an extension of the technique proposed by Mamyshev [18], spectral filtering of similariton pulses generated through distributed Raman amplification in a ND fibre has been shown to improve the ability of a $2 \mathrm{R}$ signal regenerator to eliminate spurious noise pulses as well as to simultaneously reduce any fluctuations in the signal one-bit level [19]. The frequency-chirping of signal pulses by using cross-phase modulation (XPM) with parabolic control pulses is of great interest for various high-speed processing applications where electro-optic phase modulators are not available, or cannot provide sufficient phase shift. Indeed, the chirp induced by XPM on the target pulse is proportional to the gradient of the parabolic control pulse intensity profile, so it is exactly linear. This method has been demonstrated to increase the level of tolerable timing jitter that an optical retiming system can accommodate [20]. Moreover, a near complete time-domain optical Fourier transformation can be obtained by using XPM with parabolic pulses as a time lens, and mitigation of the signal waveform distortion caused by linear perturbations in optical fibres has been successfully experimentally demonstrated with this technique [21]. A promising application of parabolic or flat-top pulses to the improvement of the signal bit-error rate in an optical communication system by using optical decision before the conventional receiver has been proposed in $[22,23]$.

The simple intensity profile of triangular pulses is also highly desired for a range of processing applications, including time-domain add-drop multiplexing [24], wavelength conversion [25], doubling of optical signals [26], and time-to-frequency mapping of multiplexed signals. Here we discuss the use of triangular pulses in the context of signal doubling and frequency conversion. A technique of copying optical pulses in both the frequency and time domains based on a combination of either XPM with a triangular pump pulse or SPM of a triangular pulse in a nonlinear Kerr medium and subsequent propagation in a dispersive medium was introduced in [26] and experimentally demonstrated in [27]. In this scheme, spectral doubling of an optical signal is made possible by the XPM or SPM phase shift generated by a temporal intensity profile with a linear gradient, which translates into a constant and distinct (opposite sign) frequency chirp induced onto the leading (down-shifted) and trailing (up-shifted) edges of the signal pulse. Under subsequent propagation of the modulated signal pulse in a linear dispersive medium, the produced spectral separation of the pulse components leads to their subsequent separation in time, thus allowing temporal replication of the pulse. Applying spectral filtering to the XPM/SPM modulated signal is also possible to realize efficient and clean frequency conversion [25, 27]. Figure 2 shows results of the experiment reported in [27]. Similarly to [13], triangular pulses were generated by passive nonlinear reshaping in a ND fibre. Spectral pulse doubling was realized through the SPM experienced by the triangular pulses over their life distance in a highly nonlinear (HNL) fibre. The output SPM-shaped spectrum from the HNL fibre was sent into either a linear standard SM fibre segment to realize temporal pulse doubling or a wavelength demultiplexer filter for frequency conversion processing.
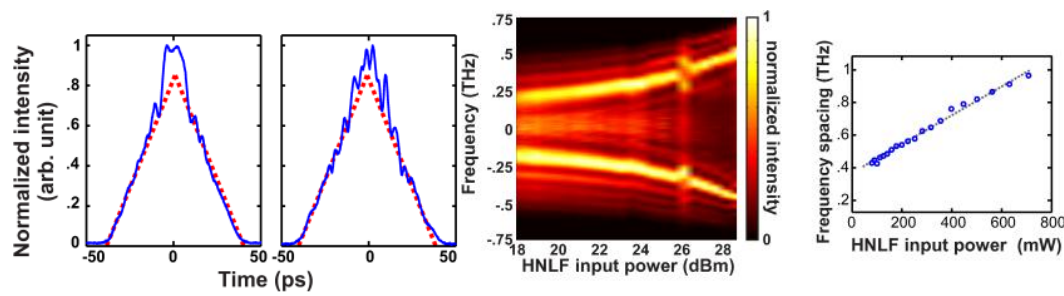

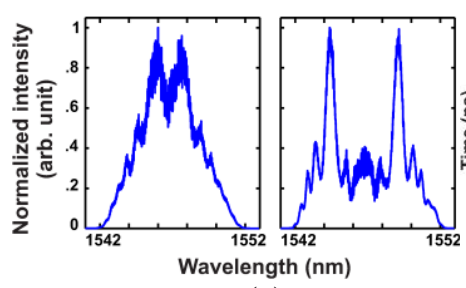

(a)

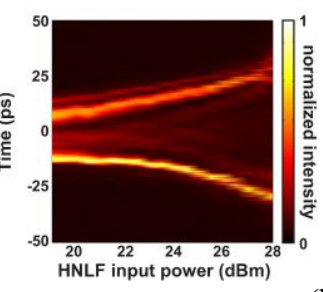

(b)

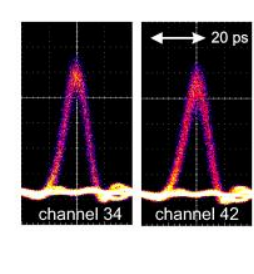

Figure 2. (a) Temporal (top) and spectral (bottom) intensity profiles after the ND reshaping fibre (left) and after the HNL fibre (right). (b) Top, evolution of the output spectrum from the HNL fibre with the input power (left), and dependence of the frequency spacing between the two spectral peaks on the input power (right). Bottom, evolution of the temporal intensity profile at the output of the linear SM fibre with the input power to the HNL fibre (left), and eye diagrams of a 10-Gbit/s pulse train obtained after spectral filtering for the ITU channels 34 and 42. Results adapted from [27]. 


\section{CONCLUSIONS}

We have provided an overview of several recent examples of the use of nonlinear phenomena in optical fibres for the generation and shaping of optical pulses. Examples of new techniques or improvements in existing techniques of all-optical nonlinear fibre-based signal processing enabled by specialized pulse waveforms have been discussed. Details and further developments of these approaches will be presented at the conference.

\section{ACKNOWLEDGEMENTS}

We thank our co-authors of the publications that form a basis for the mini-review paper presented here, and acknowledge support from the Leverhulme Trust, the EPSRC, the British Council, the Région Bourgogne, and the Ministère des Affaires Etrangères et Européennes.

\section{REFERENCES}

[1] A. M. Weiner, "Femtosecond pulse processing," Optical and Quantum Electronics, vol. 32, pp. 473-487, 2000.

[2] J. Fatome, et al., "Multiple four-wave mixing in optical fibers : 1.5-3.4-THz femtosecond pulse sources and real-time monitoring of a 20-GHz picosecond source," Opt. Commun., vol. 283, pp. 2425-2429, 2010.

[3] S. Pitois, et al., "Generation of 20-Ghz picosecond pulse trains in the normal and anomalous dispersion regimes of optical fibers," Opt. Commun., vol. 260, pp. 301-306, 2006.

[4] K. Hammani, et al., "Peregrine soliton generation and breakup in standard telecommunications fiber," Opt. Lett., vol. 36, pp. 112-114, 2011.

[5] K. Hammani, et al., "Spectral dynamics of modulation instability described using Akhmediev breather theory," Opt. Lett., vol. 36, pp. 2140-2142, 2011.

[6] M. E. Fermann, et al., "Self-similar propagation and amplification of parabolic pulses in optical fibers," Phys. Rev. Lett., vol. 84, pp. 6010-6013, 2000.

[7] C. Finot, et al., "Optical parabolic pulse generation and applications," IEEE J. Quantum Electron., vol. 45, pp. 14821489, 2009.

[8] F. W. Wise, et al., "High-energy femtosecond fiber lasers based on pulse propagation at normal dispersion," Laser \& Photonics Rev., vol. 2, pp. 58-73, 2008.

[9] C. Aguergaray, et al., "Experimental realization of a mode-locked parabolic Raman fiber oscillator," Opt. Express, vol. 18, pp. 8680-8687, 2010.

[10] T. Hirooka and M. Nakazawa, "Parabolic pulse generation by use of a dispersion-decreasing fiber with normal groupvelocity dispersion," Opt. Lett., vol. 29, pp. 498-500, 2004.

[11] C. Finot, et al., "Parabolic pulse generation through passive nonlinear pulse reshaping in a normally dispersive two segment fiber device," Opt. Express, vol. 15, pp. 852-864, 2007.

[12] S. Boscolo, et al., "Passive nonlinear pulse shaping in normally dispersive fiber systems," IEEE J. Quantum Electron., vol. 44, pp. 1196-1203, 2008.

[13] H. Wang, et al., "Generation of triangular-shaped optical pulses in normally dispersive fibre," J. Opt., vol. 12, p. 035205, 2010.

[14] B. G. Bale, et al., "Effects of fourth-order fiber dispersion on ultrashort parabolic optical pulses in the normal dispersion regime," J. Opt. Soc. Am. B, vol. 28, pp. 2059-2065, 2011.

[15] S. Boscolo and S. K. Turitsyn, "Intermediate asymptotics in nonlinear optical systems," Phys. Rev. A, vol. 85, p. 043811, 2012.

[16] J. M. Dudley and J. R. Taylor, Supercontinuum generation in optical fibers: Cambridge University Press, 2010.

[17] F. Parmigiani, et al., "Ultra-flat SPM-broadened spectra in a highly nonlinear fiber using parabolic pulses formed in a fiber Bragg grating," Opt. Express, vol. 14, pp. 7617-7622, 2006.

[18] P. V. Mamyshev, "All-optical data regeneration based on self-phase modulation effect," in European Conference on Optical Communication, ECOC'98, Madrid, Spain, 1998, pp. 475-476.

[19] C. Finot, et al., "Regenerative 40-Gb/s wavelength converter based on similariton generation," Opt. Lett., vol. 30, pp. 1776-1778, 2005.

[20] F. Parmigiani, et al., "Pulse retiming based on XPM using parabolic pulses formed in a fiber Bragg grating," IEEE Photon. Technol. Lett., vol. 18, pp. 829-831, 2006.

[21] T. Hirooka and M. Nakazawa, "All-optical 40-GHz Time-Domain Fourier Transformation using XPM with a dark parabolic pulse," IEEE Photon. Technol. Lett., vol. 20, pp. 1869-1871, 2008.

[22] S. Boscolo, et al., "Time domain all-optical signal processing at a RZ optical receiver," Opt. Express, vol. 13, pp. 62176227, 2005.

[23] I. O. Nasieva, et al., "Bit error rate improvement by nonlinear optical decision element," Opt. Lett., vol. 31, pp. 12051207, 2006.

[24] F. Parmigiani, et al., "Time domain add-drop multiplexing scheme enhanced using a saw-tooth pulse shaper," Opt. Express, vol. 17, pp. 8362-8369, 2009.

[25] F. Parmigiani, et al., "An efficient wavelength converter exploiting a grating based saw-tooth pulse shaper," IEEE Photon. Technol. Lett., vol. 20, pp. 1461-1463, 2008.

[26] A. Latkin, et al., "Doubling of optical signals using triangular pulses," J. Opt. Soc. Am. B, vol. 26, pp. 1492-1496, 2009.

[27] N. Verscheure and C. Finot, "Pulse doubling and wavelength conversion through triangular nonlinear pulse reshaping," Electron. Lett., vol. 47, pp. 1194-1196, 2011. 